\title{
Cuidados pré e pós-operatórios de queiloplastia e palatoplastia: percepção dos cuidadores em um centro especializado da região sul do Brasil
}

Pre and post-operative care of cheiloplasty and palatoplasty: perception of caregivers in a specialized center in the south region of Brazil

Cuidados pre y postoperatorios de queloplastia y palatoplastia: percepción de los cuidadores en un centro especializado en la región sur de Brasil

Maria Júlia Navarro Kassim ORCID: https://orcid.org/0000-0001-9753-2131 Universidade Estadual do Oeste do Paraná.Brasil E-mail: mjkassim_enfermagem@hotmail.com

Fabiana Gonçalves de Oliveira Azevedo Matos ORCID: https://orcid.org/0000-0002-5283-5363 Universidade Estadual do Oeste do Paraná.Brasil E-mail: fabianamatos@hotmail.com

Rejane Teixeira Coelho ORCID: https://orcid.org/0000-0002-8630-9388 Universidade Estadual do Oeste do Paraná.Brasil E-mail: enajerco@uol.com.br

Luciana Paula Grégio D'Arce Rodrigues ORCID: https://orcid.org/0000-0002-8428-5319 Universidade Estadual do Oeste do Paraná.Brasil

E-mail: lucianapgd@yahoo.com

Mariângela Monteiro de Melo Baltazar ORCID: https://orcid.org/0000-0001-6395-9516 Universidade Estadual do Oeste do Paraná.Brasil

E-mail:mmmwgb@uol.com.br Marcielle Cândido ORCID: https://orcid.org/0000-0001-6142-7399 Universidade Estadual do Oeste do Paraná.Brasil

E-mail: chelli26@hotmail.com Aline Vaneli Pelizzoni ORCID: https://orcid.org/0000-0003-1125-0200 Universidade Estadual do Oeste do Paraná.Brasil E-mail: aline.vaneli@gmail.com

Andressa Fernanda Luiz ORCID: https://orcid.org/0000-0002-2735-7224 Universidade Estadual do Oeste do Paraná.Brasil E-mail: andressa.luiz.974@gmail.com

Célia Patrícia Müller Rodrigues ORCID: https://orcid.org/0000-0002-7752-0560 Universidade Estadual do Oeste do Paraná.Brasil E-mail: pattymiiller@hotmail.com

Jeani Aparecida Petrik ORCID: https://orcid.org/0000-0002-6347-8242 Universidade Estadual do Oeste do Paraná.Brasil

E-mail: jeani.petrik@unioeste.br

Maria Yoná Silva Cabral ORCID: https://orcid.org/ 0000-0003-0781-4894 Universidade Estadual do Oeste do Paraná.Brasil E-mail: yona.sc@hotmail.com

Deisy Mery Randon Villaca ORCID: https://orcid.org/0000-0002-2610-3177 Universidade Estadual do Oeste do Paraná.Brasil E-mail: dmrandon@gmail.com

Celina Cabral

ORCID: https://orcid.org/0000-0002-9148-0227 Universidade Estadual do Oeste do Paraná.Brasil E-mail: celinacabrall@gmail.com 
Maria Grazielle Paiva Barreto Santana Lopes ORCID: https://orcid.org/0000-0001-8782-3058 Universidade Estadual do Oeste do Paraná.Brasil E-mail: grazzi_pl@outlook.com

\begin{abstract}
Resumo
O objetivo do estudo foi investigar a percepção dos cuidadores de crianças com fissura lábio palatal quanto ao procedimento cirúrgico e cuidados pós-operatórios. Trata-se de pesquisa descritiva, exploratória, com análise qualitativa dos dados realizada de acordo com a metodologia de Análise de Conteúdo proposta por Bardin. A coleta de dados foi realizada por meio de entrevista aos cuidadores durante a consulta pré-operatória multiprofissional. O instrumento de coleta de dados continha oito questões para caracterização da amostra (idade, sexo, escolaridade, renda familiar, grau de parentesco do informante, tipo de fissura, associação com síndrome genética e procedimento cirúrgico submetido) e três perguntas que buscavam explorar as dúvidas, expectativas, sentimentos e sensações relacionadas ao procedimento cirúrgico. As respostas às perguntas abertas foram gravadas e posteriormente transcritas pelos pesquisadores. Os cuidadores entrevistados demostraram dúvidas quanto ao procedimento cirúrgico e cuidados pós-operatórios e medo frente ao desfecho, no entanto, foi possível observar que havia confiança na equipe, podendo estar relacionada ao vínculo gerado desde a chegada desses pacientes ao serviço. Conclui-se que os sentimentos negativos dos cuidadores entrevistados eram gerados devido a ansiedade e as incertezas quanto ao procedimento cirúrgico. Destaca-se, portanto, a importância das orientações multidisciplinares e o estabelecimento de vínculo com pacientes e seus familiares.
\end{abstract}

Palavras-chave: Pesquisa multidisciplinar; Fenda palatina; Fenda labial; Anormalidades craniofaciais.

\begin{abstract}
The aim of the study was to investigate the perception of caregivers of children with cleft lip and palate regarding the surgical procedure and postoperative care. This is a descriptive, exploratory research, with qualitative data analysis performed according to the Content Analysis methodology proposed by Bardin. Data collection was performed through interviews with caregivers during the multiprofessional preoperative consultation. The data collection instrument contained eight questions to characterize the sample (age, sex, education, family income, degree of kinship of the informant, type of cleft, association with genetic syndrome and surgical procedure submitted) and three questions that sought to explore the doubts, expectations, feelings and sensations related to the surgical procedure. Answers to open questions were recorded and later transcribed by the researchers. The interviewed caregivers showed doubts about the surgical procedure and postoperative care and fear about the outcome, however, it was possible to observe that there was trust in the team, which may be related to the bond generated since the arrival of these patients at the service. It is concluded that the negative feelings of the interviewed caregivers were generated due to anxiety and uncertainties regarding the surgical procedure. Therefore, the importance of multidisciplinary guidelines and the establishment of bonds with patients and their families is highlighted.
\end{abstract}

Keywords: Multidisciplinary research; Cleft palate; Cleft lip; Craniofacial abnormalities.

\title{
Resumen
}

El objetivo del estudio fue investigar la percepción de los cuidadores de niños con labio leporino y paladar hendido sobre el procedimiento quirúrgico y los cuidados postoperatorios. Se trata de una investigación descriptiva, exploratoria, con análisis de datos cualitativos realizado según la metodología de Análisis de Contenido propuesta por Bardin. La recolección de datos se realizó a través de entrevistas con los cuidadores durante la consulta preoperatoria multiprofesional. El instrumento de recolección de datos contenía ocho preguntas para caracterizar la muestra (edad, sexo, educación, ingreso familiar, grado de parentesco del informante, tipo de hendidura, asociación con síndrome genético y procedimiento quirúrgico presentado) y tres preguntas que buscaban explorar las dudas, expectativas, sentimientos y sensaciones relacionadas con el procedimiento quirúrgico. Las respuestas a las preguntas abiertas fueron grabadas y luego transcritas por los investigadores. Los cuidadores entrevistados mostraron dudas sobre el procedimiento quirúrgico y postoperatorio y temor sobre el resultado, sin embargo, se pudo observar que había confianza en el equipo, lo cual puede estar relacionado con el vínculo generado desde la llegada de estos pacientes al servicio. Se concluye que los sentimientos negativos de los cuidadores entrevistados se generaron por ansiedad e incertidumbre respecto al procedimiento quirúrgico. Por tanto, se destaca la importancia de las pautas multidisciplinares y el establecimiento de vínculos con los pacientes y sus familias.

Palabras clave: Investigación multidisciplinaria; Paladar hendido; Labio leporino; Anomalías craneofaciales.

\section{Introdução}

A estimativa de indivíduos com fissura labiopalatal no Brasil é de 1:650 nascidos vivos. Tal anomalia craniofacial tem origem multifatorial, envolvendo fatores genéticos, ambientais e agentes teratogênicos (Bruggink et al., 2020). 
A fissura de lábio e/ou de palato ocorre devido falha na fusão entre os processos nasais medianos e os processos maxilares, entre a quarta e a décima segunda semana de desenvolvimento fetal, podendo ser unilateral, bilateral, completa ou incompleta (Matos et al., 2020). Diversas são as alterações morfológicas e funcionais que acometem o indivíduo com fissura orofacial, causando problemas sociais e psicológicos (Bruggink et al., 2020). Podem ocorrer distúrbios na fala, na audição, déficit nutricional, baixa autoestima, entre outros (Trettene et al., 2014).

O tratamento deve ser realizado por uma equipe multiprofissional, composta por médicos, enfermeiros, odontólogos, fonoaudiólogos, nutricionistas, psicólogos, geneticistas, fisioterapeutas e assistentes sociais, com o objetivo de garantir reabilitação física, psíquica e social do indivíduo (Cabral et al., 2021; Santos et al., 2020; Vasconcelos et al., 2020).

As cirurgias de lábio (queiloplastia) e de palato (palatoplastia) são as cirurgias reparadoras mais comuns realizadas ao longo do complexo tratamento de reabilitação. Os pacientes necessitam de um acompanhamento precoce e especializado, desde o diagnóstico da fissura até a finalização do tratamento, já na fase adulta (Moretto et al., 2017; Vasconcelos et al., 2020).

As cirurgias realizadas nos meses iniciais de vida da criança são necessárias pois garantem a correção e fechamento do lábio e/ou palato e constituem um grande desafio aos pais/cuidadores que precisam dominar um conjunto de procedimentos e encaminhamentos para a garantia de eficácia do tratamento. Estas situações desafiadoras, também mobilizam sentimentos difíceis, que podem alterar de modo significativo a saúde psicológica e causar ansiedade e estresse. Para Razera et al. (2016) as dúvidas e receios quanto ao risco operatório aumentam o aparecimento de tais sintomas.

A determinação legal sobre a criação de novos centros especializados de atendimento a pacientes com anomalias craniofaciais vem favorecendo o acesso precoce dessa clientela aos serviços de tratamento e reabilitação (Brasil, 1993; Brasil, 2002a; Brasil, 2002b). No Paraná, há três centros especializados voltados para o atendimento de pacientes com fissuras labiopalatais, sendo eles: Centro de Atendimento Integral ao Fissurado Labiopalatal (CAIF), localizado em Curitiba; Centro de Apoio e Reabilitação dos Portadores de Fissura Labiopalatal (CEFIL), localizado em Londrina; e Centro de Atenção e Pesquisa em Anomalias Craniofaciais (CEAPAC), localizado na cidade de Cascavel. Este possui habilitação em alta complexidade em Lesões Labiopalatinas pelo Ministério da Saúde (Portaria 150/2018), e atende toda a macrorregião oeste do Paraná, abrangendo 51 municípios (Baltazar et al., 2019; Kassim et al., 2021).

$\mathrm{O}$ estudo apresentado foi realizado no CEAPAC dentro do contexto da pandemia SARS-CoV-2, no qual novas práticas de saúde foram incorporadas na rotina de atendimento de saúde (Brasil, 2020). Conforme a resolução da Secretaria de Saúde do Estado do Paraná - SESA no 926/2020, que dispunha sobre a suspensão temporária da realização dos procedimentos cirúrgicos eletivos, ambulatoriais e hospitalares no Estado do Paraná, todas as cirurgias reparadoras que seriam realizadas no CEAPAC no período de março a setembro foram suspensas (Sesa, 2020a). A retomada das cirurgias eletivas no referido serviço de saúde se deu de forma gradual, respeitando a resolução SESA nº 1268/2020 (SESA, 2020b).

Diante do cenário pandêmico, foi organizado um "mutirão de cirurgia de Queiloplastia e Palatoplastia" resultante da profícua parceria entre a equipe cirúrgica do CEAPAC e equipe cirúrgica do CAIF na tentativa de atender a demanda cirúrgica represada devido à suspensão das cirurgias eletivas em decorrência da pandemia de COVID-19. Para otimizar a realização do referido mutirão, foi planejado e implementado uma intervenção multiprofissional de orientações pré-operatórias, com o objetivo de esclarecer dúvidas, prevenir complicações pós cirúrgicas e proporcionar maior segurança aos cuidadores dos pacientes após a alta hospitalar (Silva et al., 2020).

\section{Metodologia}

Trata-se de pesquisa descritiva, exploratória de natureza qualitativa, organizada a partir do mutirão cirúrgico de queiloplastia e palatoplastia realizada no CEAPAC no mês de outubro de 2020. O objetivo do estudo foi investigar a percepção dos cuidadores de crianças com fissura lábio palatal quanto ao procedimento cirúrgico e cuidados pós-operatórios. 
O estudo foi aprovado pelo do Comitê de Ética em Pesquisa da Universidade Estadual do Oeste do Paraná, CAEE no 36452320.0.0000.0107 e parecer $\mathrm{n}^{\circ} 4.250 .143$.

A coleta de dados foi realizada por meio de entrevista aos cuidadores durante a consulta pré-operatória multiprofissional. O instrumento de coleta de dados continha oito questões para caracterização da amostra (idade, sexo, escolaridade, renda familiar, grau de parentesco do informante, tipo de fissura, associação com síndrome genética e procedimento cirúrgico submetido) e três perguntas que buscavam explorar as dúvidas, expectativas, sentimentos e sensações relacionadas ao procedimento cirúrgico. As respostas às perguntas abertas foram gravadas e posteriormente transcritas pelos pesquisadores.

Após a realização da coleta de dados, os cuidadores recebiam orientações nutricionais, fonoaudiológicas, odontológicas, médicas, psicológicas, fisioterapêuticas, de enfermagem e do serviço social com base nas necessidades identificadas durante a entrevista. A abordagem era feita em ambiente adequado, seguindo as normas de segurança e distanciamento social, onde cada profissional pôde explicar questões relativas à sua respectiva especialidade.

Participaram do estudo cuidadores de seis $(85,7 \%)$ das sete crianças que fizeram parte do referido mutirão cirúrgico, sendo excluído o cuidador que não pôde participar das orientações pré-operatórias, salientando que este recebeu tais orientações em outro momento. Cabe destacar que a amostra reduzida de pacientes cirúrgicos se justifica devido às restrições impostas pela pandemia, sendo incluídos na programação cirúrgica apenas as crianças que tiveram as cirurgias suspensas pelo decreto estadual e estavam com idade limítrofe ao recomendado pelo protocolo cirúrgico de reabilitação.

Todos os participantes da pesquisa foram consultados sobre o interesse em fazer parte do estudo e assinaram o Termo de Consentimento Livre Esclarecido (TCLE).

Os dados de caracterização da amostra foram organizados a partir da sistematização das respostas das entrevistas e foram apresentados sob a forma de frequência absoluta e relativa. As informações decorrentes da transcrição das respostas dos entrevistados foram estruturadas sob a forma de unidades temáticas, permitindo a análise qualitativa dos dados por meio da metodologia de Análise de Conteúdo proposta por Bardin (2010), realizada nas seguintes etapas: organização da análise, codificação, categorização, tratamento dos resultados, inferência e interpretação dos resultados.

\section{Resultados e Discussão}

Das sete crianças incluídas no "mutirão cirúrgico", seis fizeram parte do presente estudo. Com relação à caracterização da amostra, as crianças submetidas ao procedimento cirúrgico tinham entre 10 meses a 1 ano e 10 meses de idade, com distribuição igualitária entre os sexos (50\%), sendo que 83,3\% (n=5) foram submetidos à palatoplastia e 16,7\% $(\mathrm{n}=1)$ à queiloplastia.

Quanto ao tipo de fissura das crianças, houve predominância de fissuras classificadas como transforame completa $(83,3 \%)$, sendo que 33,3\% eram fissuras bilaterais e 33,3\% eram fissuras unilaterais a esquerda. A maioria dos pacientes (83,3\%) tinha fissura isolada, sem associação com síndrome genética. Resultados semelhantes também foram encontrados nos outros estudos de Matos et al., (2020) e Rios Moura et al., (2019).

A proposta inicial da pesquisa era entrevistar os cuidadores das crianças com fissura lábio palatal a serem operadas, porém a totalidade dos cuidadores presentes durante a consulta pré-operatória multiprofissional eram mães ( $\mathrm{n}=6 ; 100 \%)$. A idade média das mães era de 26,8 anos; com escolaridade a nível de ensino médio ( $\mathrm{n}=3 ; 50 \%)$ e renda mensal de até 3 saláriosmínimos ( $\mathrm{n}=4 ; 66,7 \%)$. Resultados semelhantes foram observados em estudo anterior, onde $90 \%(\mathrm{n}=45)$ dos cuidadores eram mães, com a média de idade de 23 anos, $43 \%$ (n=23) possuíam apenas ensino médio e 62\% (n=31) eram de baixa renda (Trettene et al., 2014). 
As malformações faciais, incluindo todos os tipos de fissura, são mais frequentes em indivíduos de classe econômica baixa, estando relacionado com as restrições alimentares, não ingestão de ácido fólico e deficiência de vitaminas (Farinhas et al., 2017).

De acordo com as unidades temáticas de significação, (dúvidas, expectativas, sentimentos e sensações) as respostas das mães entrevistadas foram analisadas e organizadas em três categorias, sendo elas: "principais dúvidas dos cuidadores", “expectativas frente ao procedimento cirúrgico" e "sentimentos relativos à cirurgia".

\section{Categoria 1: Principais dúvidas das cuidadoras}

As principais dúvidas das entrevistadas relacionavam-se à dor pós-operatória, ao desconhecimento da rotina hospitalar, da técnica cirúrgica e dos cuidados com a criança após o procedimento. Tais aspectos podem ser observados nas seguintes falas:

Pl - Ah, se vai doer, tipo assim ela, se ela vai sentir dor, ou como é que vai ser.

Silveira, Lima \& Paula (2018) associam estresse e dor nas crianças hospitalizadas e identificam que as mães são precisas na sua percepção sobre a presença de dor ou não no filho. Além disso, o desconhecimento da rotina hospitalar tem implicações sobre os aspectos psicológicos, quando as informações não são suficientes e claras deixam espaço para fantasias, medos e ansiedade sobre o que pode acontecer. Na fala abaixo, a mãe expressa seu desconhecimento sobre o que irá vivenciar:

P1 - Que é a primeira vez né, eu não sei como que vai funcionar [...]

A situação hospitalar é entendida como uma experiência ameaçadora, que gera estresse, ansiedade, angústia, sentimento de inutilidade e impotência, evidenciadas com a proximidade da intervenção cirúrgica (Prudenciatti, Tavano, \& Neme, 2014; Rodrigues, Fernandes, \& Marques, 2020).

As dúvidas sobre o procedimento cirúrgico e sobre os cuidados necessários no pós-operatório, podem ser observados de acordo os excertos:

P5 - Ah, agora assim de cabeça, eu não num tenho dúvida, mas quem sabe na hora né? Porque que nem, eles vão ter que pegá e assim, como que eles vão fechá né, assim, pra mim não é mole igual a boca. Quando tinha a boca eles puxaram, então como que eles vão conseguir puxa faze, por que o palato é duro né?[...]

P6 - Então, as minhas dúvidas são assim, eu queria saber como vai ser feito essa cirurgia dela, né? Por que eu não tô fazendo idéia de como vai sê feito. Quanto tempo de duração... quantos dia vai fica no hospital, é só... quanto ao procedimento.

Percebe-se a aflição nas falas das mães acerca de como o procedimento cirúrgico seria realizado. Tal aspecto indica a necessidade de tal assunto ser abordado em algum momento do preparo pré cirúrgico, para que a família tenha conhecimento sobre a cirurgia que seu filho será submetido (palatoplastia e queiloplastia). Crepaldi (2017) referenciada em Andraus et al., (2004) destaca que, quando as pessoas dominam um sistema de informações em um determinado contexto (de ansiedade ou de dificuldade) também experimentam maior capacidade de controle cognitivo, tendo condições de manter a perturbação emocional e o estresse em níveis mais reduzidos. 
Ao possibilitar a compreensão dos aspectos envolvidos no procedimento cirúrgico, se produz uma relação de confiança entre paciente/família e equipe, que podem auxiliar na diminuição dos impactos psicológicos. Além disso, o esclarecimento das mães implica na sua participação ativa nos cuidados, influenciando significativamente na recuperação da criança (Rodrigues, Fernandes, \& Marques, 2020).

Cuidados adequados após a cirurgia irão assegurar a manutenção dos resultados, contribuindo para a prevenção de sangramento, controle da dor, alimentação, hidratação e os cuidados com a ferida operatória (Trettene et al., 2014). Sobre isso, as informações quanto aos cuidados pós-cirúrgicos apareceram como sendo uma grande preocupação das mães.

P2 - Mais é... na parte da alimentação, depois eles vão me orientar né, que é mais líquida. E a limpeza também, mais assim...

A dor pós-operatória é uma intercorrência comum nos procedimentos de queiloplastia e palatoplastia, os cuidadores devem ser orientados quanto a observação de sinais e sintomas, tais como: expressão facial, postura, choro e gemido da criança, uma vez que a comunicação verbal não é efetiva antes dos 12 meses, período em que geralmente essas cirurgias são realizadas (Trettene et al., 2014).

\section{Categoria 2: Expectativas frente ao procedimento cirúrgico}

As expectativas das mães foram, em sua totalidade, que o procedimento transcorresse bem, sem necessidade de novas abordagens e que tivessem boa recuperação. Foi possível perceber nos fragmentos de falas abaixo, a confiança depositada na equipe como uma expectativa positiva sobre o procedimento cirúrgico:

P1 - Ah! Que vai dar tudo certo né! Melhoras, a gente confia nos médicos, eles sabem que vai dar tudo certo.

P6 - [...] Então, pra mim é só expectativa boa... porque eles tratam super bem aqui todo mundo né? bem eficiente.

P6 - As melhores né, igual eu falei antes né, eu falei as melhor, porque eles são, né? São muito gente boa, tratam todo mundo bem, trabalham bem, e é.

Percebe-se que os sentimentos positivos com relação ao resultado das cirurgias resultam da confiança que é transmitida pela equipe multiprofissional. Rodrigues, Fernandes \& Marques (2020) descrevem que a desinformação e incertezas intensificam a angústia vivenciada pelos pais e justificam a necessidade de mantê-los sempre informados sobre o tratamento a ser seguido.

Cabe aos profissionais estabelecer o vínculo com o paciente e sua família desde o ingresso no serviço, o que favorecerá o processo terapêutico. A equipe multiprofissional, ao realizar acompanhamento sistemático, desempenha papel importante nas orientações aos familiares, sanando dúvidas e transmitindo tranquilidade e segurança. Para tanto, é necessário que estejam preparados para recepcionar, atender, escutar, dialogar, decidir, dar amparo e orientar (Oliveira et al., 2014).

O esclarecimento dos pais é um aspecto relevante para instrumentalizar e proporcionar estratégias favoráveis de enfrentamento, produzindo efeitos comportamentais positivos evidenciados pela autoconfiança e pela busca por suporte na resolução de problemas (Rodrigues, Fernandes, \& Marques, 2020). Tais achados podem ser percebidos nas falas a seguir:

P3 - Expectativa que ocorra tudo bem né. Que não abra nem um ponto [...].

P4 - Que seja tudo certo, que não precise fazer de novo [...] 
P5 - Eu só espero que ela dê tudo bem, tudo certo. Que não abra nenhum, nenhum pontinho né [...].

\section{Categoria 3: Sentimentos relativos à cirurgia}

A maioria das mães verbalizaram sentir medo, nervosismo, preocupação, mostrando-se ansiosas com a espera pelo procedimento cirúrgico e para saber sobre os resultados da intervenção. Tais aspectos podem ser observados nos seguintes trechos:

P1 - Ah! tô ansiosa, ansiosa... é só isso acho que eu tô muito ansiosa pra ver né o resultado.

P6 - Acho que é nervosa, né, ansiosa né, e curiosa pra vê como é que vai ficá né. Mais ansiedade de como ela vai ficá.

P4 - O meu sentimento é que não vejo a hora de fazer, sabe?! E que tudo dê certo...

Cardoso et al., (2019) revelam que o medo é um aspecto comumente identificado em pais que estão com seus filhos hospitalizados e, que este e outros elementos influenciam na qualidade de vida dos pais e na sua capacidade de atender as necessidades da criança, além de interferir negativamente no enfrentamento da situação. Os sentimentos estão, ainda, associados ao ambiente desconhecido, aos procedimentos invasivos e aos recursos internos individuais de cada acompanhante. Tais aspectos podem ser verificados nas falas a seguir:

P2 - Ah, a gente fica com medo, nervosa [...] Toda cirurgia que ele vai fazer a gente fica meio preocupado, nervosa, mas tô bem confiante que vai dar tudo certo.

P4 - ...medo todo mundo tem né, a hora que meu filho...eu fico imaginando [...].

P3 - Aaain, eu fico um pouco nervosa, mas nada assim que me abale tanto assim... eu tô tranquila, mais ou menos.

P5 - [...]porque na primeira vez eu tava muito acostumada a vê ela com o a boquinha aberta[...] e quando eu fui busca ela no centro cirúrgico eu comecei a chorá sabe, a chorá. Então agora eu tenho que começá a me prapará mais dessa vez assim porque eu tenho que ser forte pra passa pra ela, porque ela já tá mais grande, assim, ela tá mais espertinha né, então eu teria que tá mais forte pra dá mais força pra ela.

P4 - [...] eu fico imaginando, igual as mãezinhas ficam falando, dele sair perto de mim, só que entregar na mão de Deus e dos médicos que vai dar tudo certo sim.

P5 - [...] quando assim a gente vai dá ela, entrega ela, ahh (suspiro) meu Deus, é de cortá o coração, é bem triste assim, eеe eu tenho que me prepara um pouco mais [...]

O nascimento de um filho com fissura labiopalatina costuma ser uma experiência marcante e muitas vezes angustiante. Cuidadores de crianças acometidas por esta malformação podem sofrer impactos psicológicos importantes, podendo evoluir com quadros depressivos, de ansiedade e de estresse. A rotina torna-se pesada quando existe o desconhecimento quanto aos cuidados e procedimentos que os portadores de fissuras necessitam ao longo dos anos. O processo de tratamento e reabilitação cirúrgico, além de impor a necessidade de cuidados específicos, geram níveis de estresse elevado, tanto para a criança quanto para seus cuidadores, podendo culminar inclusive, na desistência do processo de reabilitação (Augsornwan et al., 2012; Razera, Trettene, \& Tabaquim, 2016). 
A rede de apoio formada por pessoas próximas aos pais é significativamente importante nesse momento, independe do tipo de ajuda oferecida, seja nos cuidados com outros filhos ou nas tarefas cotidianas, produz um sentimento de segurança e confiança necessária para vivenciarem de forma adequada a experiência cirúrgica (Menezes, Moré, \& Barros, 2016). Tais sentimentos geram efeito positivo na adaptação à situação hospitalar e operatória e, tem influência direta na adaptação das crianças que estão vinculadas emocionalmente ao adulto que o assiste (Rodrigues, Fernandes, \& Marques, 2020).

\section{Conclusão}

A partir dos resultados apresentados, conclui-se que as dúvidas das mães entrevistadas eram geradas devido a ansiedade e as aflições quanto ao procedimento cirúrgico, observa-se também que o vínculo criado com a família da criança no serviço gera maior confiança na equipe, o que apesar de não extinguir a ansiedade, aumenta a segurança de que o procedimento será bem sucedido.

Ressalta-se, portanto, a importância do preparo pré-operatório adequado, que além de sanar as dúvidas dos cuidadores quanto às necessidades fisiológicas, também acolhe a família de forma a atender suas demandas psicológicas e emocionais.

Evidencia-se a necessidade de futuras pesquisas sobre o presente tema e com amostras mais significativas, visto que no processo de levantamento bibliográfico identificou-se uma escassez de conteúdos na temática abordada.

\section{Referências}

Andrade, A. A, Rodrigues, M. C., \& Santos, W. L. (2019). A Importância da Equipe Multiprofissional para a recuperação da criança com fenda labiopalatina. Revista Enfermagem Atual in Derme. 90(28), 1-5.

Augsornwan, D. Pikhunthod, K. Pongpagatip, S., \& Surakunprapha, P. (2012). Nursing outcome in patients with cleft lip and palate who underwent operation: follow-up cases. J Med Assoc Thai. 95(11), 16-20.

Baltazar, M. M. M. Lima, D. P. Matos, F. G. A. O. Calixto, R. D. Moraes, R. S., \& Kusma, S. Z. Atenção à saúde bucal a pacientes físsurados labiopalatais no estado do Paraná. (2019). In: Rafael Gomes Ditterich, Guilherme Fernandes Graziani, Samuel Jorge Moysés (organizadores). (Org.). Caminhos e trajetórias da saúde bucal no estado do Paraná. 1ed.Londrina: INESCO, 1, 213-240.

Bardin, L. (2010). Análise de conteúdo. Ed Loyola. 229 p.

Brasil. (1993). POR: Portaria SAS/MS n. 126. Cria grupos e procedimentos para tratamento de lesões labiopalatais na tabela SIH/SUS e dá outras providências. Diário Oficial da União, 21 set 1993.

Brasil. (2002a). Ministério da Saúde. Reduzindo as desigualdades e ampliando o acesso à assistência à saúde no Brasil 1998-2002. Brasília: Editora MS. Brasil. (2002b) NO: Norma Operacional da Assistência à Saúde - NOAS - SUS 01/2002. Diário Oficial da União, 28 de fev.

Brasil. (2020) Ministério da Saúde. Painel Coronavírus.

Bruggink, R. Baan, F. Kramer, G. Claessens, C. Kuijpers-Jagtman, A. M. Bronkhorst, E. M. Maal, T. J. J., \& Ongkosuwito, E. (2020). The effect of lip closure on palatal growth in patients with unilateral clefts. PeerJ. 8:e9631

Cabral, C. Lopes, M. G. P. B. S. Oliveira, D. L., \& Baltazar, M. M. M. (2021). Abordagem fonoaudiológica em pacientes com fissura labiopalatal em serviço especializado de alta complexidade na região oeste do Paraná. RSD Journal. 10(10), e144101019062.

Cardoso, T. P. Oliveira, P. R. Volpato, R. J. Nascimento, V. F. Rocha, E. M., \& Lemes A. G. (2019). Vivências e percepções de familiares sobre a hospitalização da criança em unidade pediátrica. Rev. Enferm. UFSM, 9(4), 1-22.

Farinhas, G. V. Mattje, A. V. Polonio, D. F., \& Garcia, E. L. (2017). Sobre mães e seus cuidados para com filhos acometidos por malformação labiopalatal. Jornada de Pesquisa em Psicologia. 1-14.

Kassim, M. J. N. Matos, F. G. O. A. Cândido, M. Borges, G. S., \& Rodrigues, L. P. G. D. (2021). Consulta de enfermagem a pacientes com fissuras labiopalatais. Revista Eletrônica Acervo Saúde, 13(4), e6992.

Matos, F. G. O. A. Santos, K. J. J. Baltazar, M. M. M. Fernandes, C. A. M. Marques, A. F. J., \& Luz, M. S. (2020). Epidemiological profile of labiopalatine clefts in children cared for at a reference center in Paraná. Rev. Enferm. UFSM - REUFSM Santa Maria, RS,10(28), 1-15.

Menezes, M. Moré, C. L. O. O., \& Barros, L. (2016). As redes sociais dos familiares acompanhantes durante internação hospitalar de crianças. Rev. Esc Enferm USP, (50), 107-113.

Moretto, M. J. Fernandes, S. L. Lemes, A. T. A. L. Ferreira, E. S., \& Sales, C. F. R. (2017). Tratamento multidisciplinar na reabilitação de pacientes portadores de fissura de lábio e/ou palato. J Multidiscipl Dent, 10(1), 3-8. 
Research, Society and Development, v. 10, n. 13, e515101321511, 2021

(CC BY 4.0) | ISSN 2525-3409 | DOI: http://dx.doi.org/10.33448/rsd-v10i13.21511

Paraná. Resolução SESA N ${ }^{\circ}$ 1268/2020, 13 de setembro de 2020. Regulamenta o disposto nos arts. $1^{\circ}, 2^{\circ}, 3^{\circ}, 10,13$ e 15 do Decreto Estadual no $4.230,16$ de março de 2020, para implementação e manutenção das medidas de enfrentamento à COVID-19.

Paraná. Resolução SESA No 926/2020, 24 de julho de 2020. Dispõe sobre a suspensão temporária da realização dos procedimentos cirúrgicos eletivos ambulatoriais e hospitalares, em face da escassez de medicamentos anestésicos e relaxantes musculares no Estado do Paraná.

Razera, A. P. Trettene, A. S., \& Tabaquim, M. L. M. (2004). O impacto estressor das cirurgias primárias reparadoras em cuidadores de crianças com fissura labiopalatina. Bol. Acad. Paulista de Psicologia. 36 (90), 105-123.

Rossato, L. M. (2004). Dimensões do cuidado da criança com dor e de sua família. Rev. Min. Enf, 8(4), 501-505.

Santos, J. V. N. Tavares, J. L. F. Silva, M. A. B. Cavalcanti, A. F. M. Barbosa, D. N., \& Leite, R. B. (2020). Fissura labiopalatina: estudo do papel do profissional de saúde na diminuição de danos ao paciente. Revista Ciencia E Odontologia, 4(1), 48-55.

Silva, N. F. Beluci, M. L. Banhara, F. L. Henrique, T. Manso, M. M. F. G., \& Trettene, A. S. (2020). Dúvidas de pacientes e cuidadores informais relativas aos cuidados pós-operatórios de enxerto ósseo alveolar. Rev. Bras Enferm, 73(5).

Trettene, A. S. Mondini, C. C. D. S., \& Marques, I. L. (2013). Feeding children in the immediate perioperative period after palatoplasty: a comparison between techniques using a cup and a spoon. Rev Esc Enferm USP,47(6),1298-1304.

Trettene, A. S. Razera, A. P. R. Maximiano, T. O. Luiz, A. G. Dalben, G. S., \& Gomide, M. R. (2014). Dúvidas de cuidadores de crianças com fissura labiopalatina sobre os cuidados pós-operatórios de queiloplastia e palatoplastia. Rev. esc. enferm. USP. São Paulo, 48(6).

Vasconcelos, B. B. N. Albuquerque, D. F. Cavalcante, R. B. Teixeira, R. C. Neto, F. P. V. B., \& Ferreira, M. M. (2020). Qualidade de vida de pacientes acometidos por fissuras labiopalatinas sob a visão do cuidador. Braz. J. of Develop. Curitiba, 6(7) ,47807- 47821.

Volpato, B. C., \& Crepaldi, M. A. (2017). Percepções das mães sobre a preparação pré-cirúrgica de seus filhos segundo dois modelos. Psicologia Argumento, 29(66). 